\title{
Premier aperçu sur les cultes des Soninké émigrés au Mande
}

A first look at the cults of Soninke who emigrated to Mande

\section{Germaine Dieterlen}

\section{(2) OpenEdition}

12 Journals

Édition électronique

URL : http://journals.openedition.org/span/93

DOI : $10.4000 /$ span.93

ISSN : 2268-1558

Éditeur

École pratique des hautes études. Sciences humaines

\section{Édition imprimée}

Date de publication : 1 septembre 1975

Pagination : 5-18

ISSN : 0294-7080

\section{Référence électronique}

Germaine Dieterlen, «Premier aperçu sur les cultes des Soninké émigrés au Mande », Systèmes de pensée en Afrique noire [En ligne], 1 | 1975, mis en ligne le 02 juillet 2013, consulté le 30 avril 2019. URL : http://journals.openedition.org/span/93; DOI : 10.4000/span.93 
PREMIER APERCU SUR LES CULTES DES SONINKE EMIGRES AU MANDE

par Germaine DIETERLEN

RESUME - A la suite de la diaspora qui suivit la chute de l'Empire du Ghana les Soninké émigrèrent vers le sud et peuplèrent notamment les régions riveraines du Niger.

Implantées très anciennement dans la région du Mandé, certaines familles soninké, relevant du clan Kagoro, y avaient transporté leurs cultes en même temps qu'ils devenaient "maitres de la terre" dugu kolo tigi. Ces divers cultes, kpnq, bqmba, sqda, etc.. sont encore en viqueur aujourd'hui; ce sont eux qui contribuent à affermir la cohésion sociale et la solidarité économique qui lient les membres de ces diverses familles, et à leur conserver leur spécificité, bien qu'elles aient été intégrées, sous le règne de Soundiata, aux groupes ethniques relevant de l'empire du mali.

Nous apportons aujourd'hui, recueillies auprès de wâ Kamissoko avec le concours de Youssouf Tata Cissé, les premières informations sur certains de ces cultes.

Les guerres, la sécheresse, les risques de famine et l'épuisement des mines d'or ont contraint les. Soninké (1) de l'empire de ouagadou, qui peuplaient le Sahel, à émigrer par vagues successives à des époques diverses, vers le sud, sur les rives du Niger et notamment dans la région du Mandé. Lorsque les familles soninké relevant du clan Kagoro l'ont occupée et y ont apporté leurs cultes, cette région était déjà partiellement occupée par les Traoré (2).

Une fraction importante de cette dernière famille, émigrant du Ouagadou se serait tout d'abord installée à Somadougou, dans la région de Mopti où elle aurait édifié le premier kamã bir.

(1) Une bibliographie exhaustive sur les Soninké a été êtablie par E. Pollet et G. Winter et publiée dans le Journal de $2 a$ Société des Africanistes tome XXXIV fasc.2 p.283 à 292, ainsi que dans leur ouvrage sur La Société Soninké (Dyahunu - Mali). Edition de l'Institut de Sociologie de l'Université Libre de Bruxelles, 1971 ; p.531 à 537.

(2) Notamment les monts du Gouangaran, au S.O. de Kita.

Les généalogistes font remonter la première migration des Soninké vers le sud, conséquence de ce qu'ils nomment la première destruction de l'Empire de Ghana, à plus de 1500 ans avant notre ère. Elle fut suivie de quatre autres qui s'échelonnent jusqu'à la fin de l'Empire de ouagadou. Ces traditions sont à l'étude. 
Ce culte aurait été transporté et diffusé au fur et à mesure de leurs déplacements vers l'est, dans les régions de San puis de ségou où il existe encore plusieurs kamãa bló, enfin au Mandé où les Traoré implantèrent le premier sanctuaire à Kinièro. Minkongo, considéré comme l'un des plus anciens villages de la région de Bamako, fut fondé par Makan Sikoro Traoré, venu avec les siens de Balanzan. Ce sont les membres de cette agglomération qui ont réalisé les peintures des abris sous roche de Koulouba. Un de leurs ancêtres, Dyola Traoré, était devin. Il exécutait ce travail avec 17 cailloux de latérite qu'il jetait sur le sol et dont la position dictait les sacrifices à effectuer. Il reproduisait ensuite les figures ainsi formées en ocre rouge sur les parois des cavernes. Elles s'appelaient saw ou sao, terme que l'on traduit par "lecture rapide" ; on disait d'elles aussi

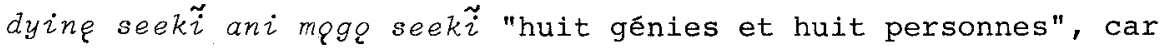
elles étaient assimilées à 16 figures géomantiques.

Les Traoré étaient des chasseurs ; cependant ils cultivaient le maiss et le fonio "blanc". Les nouveaux arrivants étaient des cultivateurs de petit mil et de sorgho au Ghana. A la suite de tractations, ils devinrent maittres de la terre cultivée, les Traoré restant maîtres de la brousse.

Après l'occupation des lieux par les Konaté, qui prirent sous le règne de Soundiata le nom de Kéita, les Traoré perdirent leur autonomie politique. Ils ont conservé les kamã blõ de Kiniéro et de certaines agglomérations de Guinée; les Kéita édifièrent celui de Kangaba (Kaba) et assumèrent la responsabilité du culte (1)

Les familles soninké relevant du clan Kagoro, implantées au Mandé, sont dites Blaw; 5 d'entre elles avaient exercé le commandement sur la Kagoro au Ghana : Kamara, Kamissoko, Doumbia (ou Sissoko), Bagayogo (ou Sinayogo) et Danyogo (2).

(1) Il conviendrait de faire une étude approfondie des migrations anciennes des Traoré, de leurs implantations et des cultes qu'ils ont instaurés dans le Mandé.

(2) Sur ces nom de famille voir : Humblot. Du nom chez les Malinké. Bulletin du Comité d'Etudes historiques et scientifiques de $1^{\prime} \mathrm{AOF}, \mathrm{n}^{\circ} 18 \mathrm{p} .526$ Note 1 ; Ch. Monteil. Les Empires du Mali. Maisonneuve et Larose. Paris 1968, p.22 et 26. cet auteur fait des Boula des affranchis ayant "joué un grand rôle dans toutes les formations indigènes". 


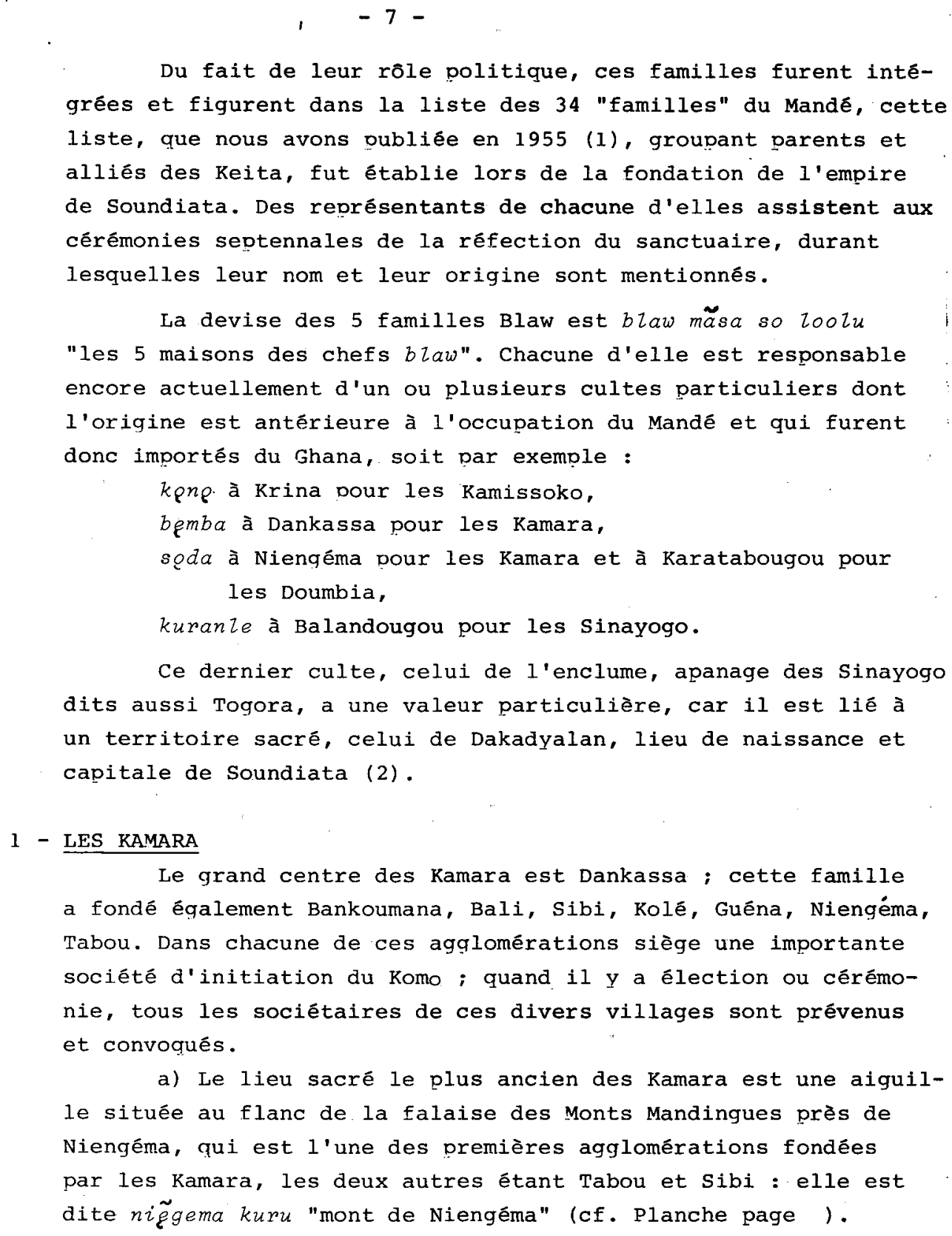

Du fait de leur rôle politique, ces familles furent intégrées et figurent dans la liste des 34 "familles" du Mandé, cette liste, que nous avons oubliée en 1955 (1), groupant parents et alliés des Keita, fut établie lors de la fondation de l'empire de Soundiata. Des représentants de chacune d'elles assistent aux cérémonies septennales de la réfection du sanctuaire, durant lesquelles leur nom et leur origine sont mentionnés.

La devise des 5 familles Blaw est blaw mãsa so loolu "les 5 maisons des chefs blaw". Chacune d'elle est responsable encore actuellement $d$ 'un ou plusieurs cultes particuliers dont l'origine est antérieure à l'occupation du Mandé et qui furent donc importés du Ghana, soit par exemple :

kenp. à Krina dour les Kamissoko, bemba à Dankassa pour les Kamara, sqda à Niengéma pour les Kamara et à Karatabougou pour les Doumbia, kuranle à Balandougou pour les sinayogo.

Ce dernier culte, celui de l'enclume, apanage des sinayogo dits aussi Togora, a une valeur particulière, car il est lié à un territoire sacré, celui de Dakadyalan, lieu de naissance et capitale de Soundiata (2).

1 - LES KAMARA

Le grand centre des Kamara est Dankassa; cette famille a fondé également Bankoumana, Bali, Sibi, Kolé, Guéna, Niengéma, Tabou. Dans chacune de ces agglomérations siège une importante société d'initiation du Komo ; quand il y a élection ou cérémonie, tous les sociétaires de ces divers villages sont prévenus et convogués.

a) Le lieu sacré le plus ancien des Kamara est une aiguille située au flanc de la falaise des Monts Mandingues près de Niengéma, qui est 1 'une des premières agglomérations fondées par les Kamara, les deux autres étant Tabou et Sibi : elle est dite nig̈gema kuru "mont de Niengéma" (cf. Planche page).

(1) G. Dieterlen. Mythe et organisation sociale au Soudan français. Journal de la Société des Africanistes. T XXV 1955 p. 41. Mythe et organisation sociale en Afrique Occidentale. Journal de la Société des Africanistes. T XXIX 1959 p. 119 à 138 .

(2) Y. Cissé et wâ Kamissoko - Histoire de l'Emoire du Mali (ronéo), colloque sur les traditions orales. Bamako, mars 1973. Tome 1 p. 79 - Tome II p. 112. 


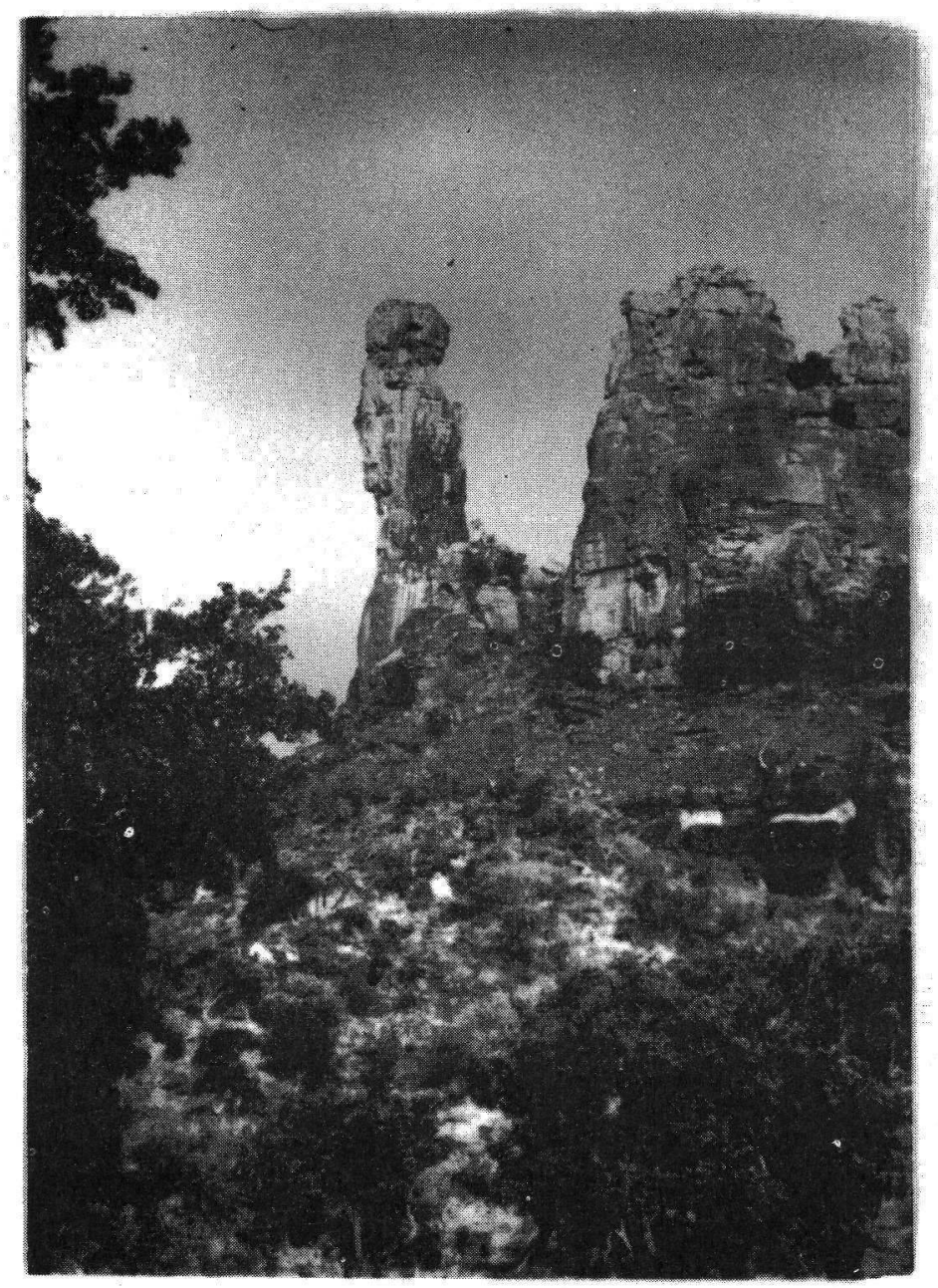

L'aiguille de Nienguéma

(Cliché Jean Rouch) 
Un prêtre, initié depuis l'âge de 15 ans environ, exécute seul un sacrifice annuel pour la fertilité du sol et la fécondité du groupe, la vie sociale, économique, politique, comme autrefois Lors des querres. Ce prêtre est dit wara sa, litt. "lion-serpent". or ce terme désigne également un oiseau identifié, il connote donc trois catégories d'animaux.

Le wara sa est choisi pour ses qualités décelées dès l'enfance. Après les rites de sortie de la circoncision, on enferme les enfants dans une case dont on obstrue les issues et d'ou on leur demande de sortir. Ceux qụi réussissent sont considérés comme de vrais thaumathurges, suba ; ils hêritent, dit-on, de leur mère le don d'agir. Cette épreuve est effectuée encore aujourd'hui à Krina et dans d'autres villages de fondation. On procède ensuite à un choix parmi les Kamara qui ont triomphé de l'épreuve ; celui qui est désigné peut relever de n'importe quel village, les bâtards étant seuls exclus. Il reçoit immédiatement une instruction particulière, car il doit dès sa nomination respecter tous les interdits du lieu. Il est ensuite l'objet d'une intronisation secrète, semblable. à celle que subit le prêtre du kono de Krina. Le wara sa ne consomme jamais les céréales de l'année sous quelque forme que ce soit ; sa bière est faite avec le mil des années précédentes. Son repas est préparé la veille. Mais il peut siéger partout où se trouve le komo et il peut vivre dans son propre village.

prêtre sacrificateur, le wara sa est "voyant", comme l'est aussi le chef. Il récite une formule et, à l'état de veille ou en rêve, effectue un "voyage mystérieux et révélateur". Il doit donc posséder et exercer des dons chamanistiques.

Le sacrifice a lieu après la récolte de deux variétés natives de riz et de sorgho. L'officiant verse un mélange d'eau et de farine crue au sommet de l'autel-piton 3 ou 4 mois après les semailles, le premier dimanche du mois, ou le dernier si les céréales ne sont pas mûres.

b) A Dankassa, le culte de bgmba (litt. : entente), est considéré comme le "jumeau du culte du kqn̨ (litt. : oiseau) de Krłna dont sont responsables les Kamissoko de cette localité, le premier étant "mâle" et le second "femelle". 
Deux dignitaires sont affectes au culte de bemba, un pretre le marê et une pretresse, la maremba. La pretresse est toujours la fille ainee des autochtones de Dankassa. Obligatoirement chaque homme originaire du Dankassa doit donner en marlage sa première fille à un homme résldant dans le village. Automatiquement, la plus agé d'entre elles assume les fonctions, perd son patronyme et prend le nom de maremba. Mère, $b a$, du pretre, marế, elle est considerée comme la mère du village tout entier.

Le pretre, marê, est toujours celui qui vient en âge immédiatement après le chef du village. Si le chef meurt, on n'intronise pas un autre marế, c'est celui qui le suit en ăge qui devient chef. Ce pretre subit une castration lors de son intronisation dite marế lasigi "asseoir le marê". Le sang qui coule de la plaie tombe dans un trou creusé dans le sol; la terre sanglante mélangée a du charbon sert à soigner la plaie. L'intronisé consomme immédiatement un jeune chien grillé assaisonné de sel et de piment. Cette castration a une valeur symbolique où intervient la présence des "elements" de base (1).

Deux champs de fonction sont associés au culte de $b_{g} m b a$ : l'un de petit riz "noir" mazo le, l'autre de sorgho "blanc" kqnde, qui doivent être contigus, le champ de sorgho devant être situe un peu au dessus de l'autre. Le produit des récoltes est utilisé l'année suivante pour les cérémonies, le reste revenant exclusivement au chef.

Rite annuel. Avant l'aube du jour choisi, vers $4 \mathrm{~h} 1 / 2$ du matin, 7 officiants se rendent derrière le village et effectuent les sacrifices sur l'autel bęmba, transporté pour la circonstance depuis le sanctuaire qui lui est affecté dans le village, enveloppé dans un tissu, posé dans une calebasse placée dans un filet.

Le sacrificateur passe le reste de la nuit les mains derrière le dos, assis, la jambe gauche repliée, l'autre dressée.

La viande est cuite sur place par la maręmba qui a apporté les ustensiles de cuisine.

7 des officiants consomment la viande de $b_{q m b a}:$ le chef, le prêtre et les 5 hommes les plus âgés du village. Vers $2 \mathrm{~h} 1 / 2$ de 1 'après-midi, les musiciens viennent à leur rencontre, suivis de tous les villageois et du masque ndomo porté par un jeune garçon. La prêtresse qui a préparé les aliments ramasse les

(1) Voir plus bas p. 12 
ustensiles qui ont servi à la cuisson du repas. On charge bqmba sur la tête du marẽ qui marche précédé de la maręmba. Derrière le porteur, un seul tambourinaire joue du tambour d'aisselle. On chante. Tous les autres instruments se règlent ensuite sur le tambour et tous les participants font 7 fois le tour du village, passent ensuite par toutes les rues, puis vont à la place publique dont il faut aussi faire 7 fois le tour en procession, en chantant un chant qui exalte les vertus guerrière des kamara : "- eh ! chaude fournaise (bis) les jeunes Kamara à eux seuls constituent une armée

- eh ! chaude fournaise (bis) chaude fournaise de la guerre, les descendants de Kaman Dyan constituent à eux seuls une armée

- eh ! chaude fournaise (bis) chaude fournaise de la guerre, les descendants de Fakoli constituent à eux seuls une armée (1)".

On raccompagne ensuite chez lui le chef du village, puis le prêtre ainsi que l'autel bemba qui sera réintégré dans le sanctuaire, puis la prêtresse, enfin les autres officiants.

Le marẽ dépose bęmba dans le sanctuaire, entre à reculons et en sortira de la même façon; il place bemba sur un support élevé fiché au sol par 4 pieds. Il allume dessous un petit feu qu'il devra entretenir toute l'année; le bois provient obligatoirement d'un arbre fouaroyé.

De son côté, lorsque la prêtresse arrive chez elle, elle s'agenouille et remet sa charge à une fillette; une vieille femme l'aide à décharger les ustensiles. La maręmba va ensuite puiser de l'eau dans une jarre neuve qu'elle place dans sa maison; avec deux cuillers-calebasses qu'elle tient dans chaque main, elle puise l'eau de la jarre, croise ses bras et, dans cette position, donne le contenu de la cuiller de gauche au marẽ qui est venu la rejoindre et boit celui de la cuiller de droite. Les eaux restantes sont ensuite mises dans une calebasse neuve. Au premier chant du cog, le lendemain, la maremba, seule en cachette, ira verser cette eau dans le fleuve. Après quoi, elle brisera les calebasses et les jettera au fleuve avant de rentrer au village, car personne ne doit toucher à ces récipients.

(1) e wombe, e wombe (ou womba) kamara denu ganza ye kele ri $e$ wombe, e wombe kele wombe, kele wombe, kamã-dya si gaza ye kezle pi $e$ wombe, e wombe, kele wombe, fakoli si ganaa ye kqlq ri 
Pendant que la maremba offre l'eau au marẽ, les assistants chantent le dyãayõ qui relate l'origine des 5 familles blaw (1).

Les participants se rendent ensuite sur la place publique où sera consommé un repas communiel, de la bière et de l'hydromel. Tous dansent ensuite jusqu'au lever du soleil sur des rythmes anciens joués sur des tambours et instruments à cordes. Les diverses séquences du rituel soulignent la restauration effectuée dans le culte de bemba par la présence des 4 éléments de base. En effet, la maremba est associée à l'élément "eau", source de toute vie; le marê au "feu" entretenu toute l'année; les deux autres éléments sont rappelés par la castration subie : le trou qu'il a creusé lors de son intronisation - soit "l'air" - dans la "terre" qui a reçu son sang.

Le culte de bemba est le fondement de l'autorité religieuse, sociale et politique des familles Kamara.

\section{2 - LES SINAYOGO}

Les Sinayogo sont les responsables d'un autel portatif dit kurâa $l_{e}$ "petite enclume". Il n'existe qu'un seul exemplaire de cet autel, qui est toujours placé sous la garde du plus âgé des chefs de lignages de cette famille. Il peut donc être amené à changer de lieu d'affectation après la mort du responsable (2).

Le culte de cette enclume vaut pour l'ensemble du groupe ethnique vivant au Mandé et pour tout ce qui le concerne. Nous donnons ci-après le rite très ancien qui a présidé à la confection et à la consécration de cet autel.

L'autel-enclume, kurâa le, est constitué d'une tige d'or (sanu), d'une tige de cuivre rouge (danya) et d'une tige de cuivre jaune (suza), enfermées dans une feuille de fer noir (nqgq $f^{\tilde{i}}$ ) L'ensemble est battu, martelé à chaud et trempé. On confectionne ainsi une enclume qui comprendra les 4 éléments de base symbolisés respectivement pour le "feu" par l'or, "l'eau" par le cuivre jaune, "l'air" par le cuivre rouge, la "terre" par le fer.

(1) Sur le dyãaỹo, hymne qui exalte "les vertus des chasseurs et la marche épique des grands hommes du Manding", soir Y. Cissé. "Un récit initiatique de chasse ou les exploits d'un chasseur légendaire". Diplôme de 1'EPHE, $5^{\circ}$ Section, p. 76 et 280 (ronéo). Voir également Y. Cissê et Wâ Kamissoko loc. cit. p. 58 et suivantes.

(2) Après avoir été détenu par N'Fa Lamine Sinayogo de Balandougou, , I'autel est actuellement sous la garde de Massama Sinayogo de Koukola, hameau situé près de Balandougou. 
L'or est à la base car il représente la pureté et l'incorruptibilité. Plus il vieillit, plus l'or a de force. Son témoin est le cuivre rouge; c'est le support de la puissance agressive et dangereuse, il est "chaud et ardent". Le cuivre jaune est utilisé pour guérir, ou transmettre les maladies. Avant d'intégrer cette tige dans l'autel, on la fiche dans le tronc d'un ficus. L'officiant monte jusqu'au farte et verse sur les feuilles le lait d'une génisse première née; puis là où est tombé le lait, on creuse pour extraire du sol un morceau de racine : son écorce mêlée à d'autres ingrédients (venins de scorpion, de serpent, produits corrosifs, ainsi que de nombreuses variétés de céréales et de végétaux de brousse) mis dans l'eau servira à confectionner un korte (1) dit sula la dyigĩ ("cuivre descendu") qui sera utilisé pour 1 'exécution des coupables. L'officiant prend ensuite le morceau de cuivre jaune planté dans le ficus pour l'intégrer à 1 'autel.

Pendant la fabrication, on récite le texte suivant : $n^{\prime} y^{\prime} i s \alpha i k o$ sa dyo "je t'ai acheté comme on achète une esclave" $n^{\prime} y^{\prime} i$ furu $i$ ko furu dyo "je t'épouse comme on épouse une esclave" $k a i$ kanu $i$ ko kanu dyo "et je t'aime comme on aime une esclave de l'amour".

L'enclume est accompagnée d'un petit marteau de fer qu'on appelle "le petit de l'enclume", confectionné en fer noir provenant d'un haut fourneau. On le coiffe d'un oeil gauche humain attaché avec un nerf humain prélevé à gauche du sacrum, qu'on laisse sécher. L'oeil et le nerf proviennent d'une vierge décédée. L'oeil est le symbole, le reflet de la personne; le nerf du sacrum, à cause du bassin, en est le fondement. La jeune fille représente kakun ga "la grande kakun, personnalité mythique dite "mère des choses anciennes".

Cet objet a la longueur a'une palme, du pouce à l'auriculaire et l'enclume la hauteur d'une main. Ces deux objets sont consacrés par un sacrifice effectué en présence des seuls sinayogo dans le bosquet sacré du komo (dit gwé tu, touffe de la pureté"). On les place dans un morceau de poterie cassée au dessus de laquelle on égorge successivement un coq noir, un coq rouge, un bouc, un chien rouge. Les coqs rouge et noir représentent toutes les variétés d'êtres humains; le bouc est le symbole

(1) Sur les korte cf. G. Dieterlen. Essai sur la religion Bambara p. 147 . 
de la virilité et de la fëcondité; le chien rouge est le témoin de la parole, du verbe, du rhombe.

Les participants retournés au village, le kurä tigi et son sacrificateur cuisent et consomment seuls sur place les volailles sacrifiées.

On laisse les objets dans le bain de sang pendant 8 jours, le 8 ème jour, à midi, on les retire. Ils sont devenus des "dyo", la complétude du temps venant s'ajouter à la complétude des éléments. Avant midi, le masque du kqme était venu tourner en hurlant autour du tertre, tandis que les sociétaires présents chantaient le chant des forgerons.

Dans l'agglomération, à partir de deux heures de l'après midi, tous les sinayogo des deux sexes chantent et dansent jusqu'au crépuscule, les hommes brandissant des haches, les femmes des vans. Le soir venu, on sonne les trompes du kqm? : les femmes et les non-initiés se retirent. Après le repas du soir, les sociétaires et les membres du kpmq voisin reconduisent au village le chef du kura le qui porte sur la tête les objets consacrés dans le tesson de canari. On les dépose dans le sanctuaire, sur une petite étagère à claire-voie, placée sur le sol. On ferme le sanctuaire, on appuie une hatte de paille contre la porte et on y plante le couteau sacrificiel à un seul tranchant (da kezer) qui a été également fait avec du fer provenant d'un haut fourneau.

Tous les ans, les responsables effectuent le sacrifice d'un coq noir et d'un coq rouge, au premier jour de la lune dit dyõ mqne, suivi d'une beuverie de bière de sorgho blanc (kende). Les Sinayogo, autrefois chefs de guerre, ont abandonné la métallurgie ; mais, à l'origine, ils étaient forgerons.

3 - LES DOUMBIA

Les Doumbia installés au Mandé descendent pour la plupart de trois frères, originaires de Farakoba, site historique où se seraient séparées les familles blaw émigrées de ouagađou. Ces trois frères, Koliyoro, Tamba et Fotigui ont fondé Karatabougou, agglomération où se trouve le sanctuaire consacré au sqda. Actuellement, cette famille occupe au Mandé sept villages dont les membres se groupent pour célébrer ensemble le rite annuel et le sacrifice offert au sqda: ce sont Karatabougou, Touréla, Sonkoréla, Niènkènkoro, Sanankoro, Kori et Kandyan. Le sanctuaire est constitué d'une habitation circulaire sans porte, couvrant 
deux profondes excavations dites "puits", respectivement minimini $k_{q} z_{q}$ "puits du tournoiement" et $f a$ bali $k_{q} i_{\tilde{q}}$ "puits qui ne se remplit pas" ou "sans fond". Le premier connote le "tournoiement de la création et de l'univers" et, sur le plan historique, les déplacements des Soninké. Le second, qui contient tout ce qu'on a pu y mettre en miniature, à titre de témoin (matières animales, végétales, minêrales, petits objets etc...) connote cet univers "inêpuisable" et la vie "immortelle" qui l'anime. A l'intérieur, sur les parois de ce sanctuaire qui est dit "voix de l'avenir" sinin $k \tilde{a}$ sont peints les "signes" sacrés, comme on le fait sur celles du sanctuaire du Mandé de Kangaba.

Au jour dit, chaque année, un délégué par village portant une volaille, des noix de cola et du sorgho se rend à Karatabougou : on sacrifie sur les deux puits trois poulets blancs et quatre noirs (offerts respectivement les blancs pour les "paroles" l'entente, le bonheur de la collectivité, les rouges pour parer à tous les dangers, guerres, famines, épidémies, etc..) du "lait" de mil étant en même temps pour la fécondité. Les trois fonaateurs avaient en ce lieu fait le serment de ne jamais se séparer et marqué ainsi la prise de possession du territoire qui, à l'époque, n'était pas occupé.

\section{3 - LES KAMISSOKO ET LES. KEITA OU MASARE}

Les Kamissoko, qui auraient quitté le Sahel lors de la première et très ancienne sécheresse qui provoqua la première diaspora, ont occupé successivement : Tona, Tarakouma, Kaya, Nanéko, Balaninko, Fouyan, Dyondyougouré, Dyombané, Fanifasimbo, Fanifa Marinyouman, Banankoro, Somabogoriman, Koroninflé et Krina. Contrairement à d'autres groupes de migrants ils ne sont jamais retournés vers le Nord. Il y a actuellement des membres de cette famille portant la devise Déréba, dans la région de Ségou (1).

Une première documentation a été recueillie sur le culte de "l'oiseau" kqnq de Krina dont sont reposables les Kamisoko et qui est considéré, nous l'avons vu comme le "jumeau de bqgmba";

(1) dereba est une devise donnée autrefois aux individus très instruits et compétents. 
de nouvelles recherches ont été entreprises sur le sanctuaire de Kangaba, kamã bló, et sa réfection septennale. Ces informations, qui font l'objet d'un article en préparation, complètent les enquêtes consignées dans nos publicaticns artérieures (1).

Ce premier aperçu sur certains cultes soninké du Mandé ne permet pas actuellement de dégager ie lien qui serait susceptible de les unir. Il se peut que chacun d'elix, assoclé à une famille, s'insère encore actuellement, à un certair niveau, dans un ensemble cohérent propre à l'ethnie, ensemble dont il conviendra de dégager les structures sociales et religieuses. Mais il conviendra également d'examiner l'histoire, la diffusion de ces cultes après la diaspora, leur implantation actuelle, puis de dégager les influences subies par le contact avec d'autres groupes ethniques comme avec l'islamisme et les modifications qui en ont résulté.

En effet, au cours de cette enquête, nous avons également recueilli des informations sur les migrations des soninké postérieures à leur installation dans le Mandé, et le peuplement de diverses régions d'Afrique Occidentale, informations qui confirment et complètent celles qui ont été publiées dans divers articles traitant de ce sujet (2).

Nous donnons ci-après celles qui concernent l'occupation des falaises de Bandiagara.

On sait que lorsqu'un groupe émigre il transporte souvent avec lui une portion de la terre d'origine (3). Il donne parfois aussi au lieu oũ il s'installe le nom de l'agglomération d'où il vient ; un lieu de culte ou un autel peut également porter ce nom. Un patronyme ou un toponyme sont donc parfois rêvélateurs de l'origine des occupants.

(1) cf. supra p. $3 \mathrm{n} \cdot 3$

(2) Notamment, Contribution à l'étude des relations protohistoriques entre le Mandé et l'ancien Ghana, in Actes du Symposium international sur les Religions de la Préhistoire, Valcamonica, septembre 1972, sous presse.

(3) Ainsi le culte du Lebé, rendu par les quatre "tribus" dogon à la "terre" du Mandé qu'ils avaient transportée avec eux lors de la migration cf. S de Ganay, "Note sur le culte du Lébé chez les Dogon" Journal de la Société des Africanistes, tome VII, 1937, p. 203 à 212 . 
Wéréwéré Suleymane Konaté $n$ 'a pas voulu se convertir à l'islamisme à l'époque où soundiata accédait au pouvoir. Ce dernier l'avait envoyé au sénégal acheter des chevaux. Aprẽs son retour, il a quitté le Mandé avec sa famille, ses biens, ses autels et ses "secrets"; gundo ou gyindo. Il a dirigé l'exode et ses descendants. se sont fixés dans les falaises de Bandiagara. Gyindo est le terme malinké ancien pour "secret, mystère, connaissance secrète" ; or, c'est la devise que portent encore actuellement tous les Dogon. Les gyindo sont des Masaré, car le fondateur était le demi-frère de Soundiata. Il était géomancien, possédait un "bâton" (gerene) utilisé pour la divination, questionnait l'oryctérope et le renard. Le procédé est dit timba kinye,"sable de l'oryctérope". Aujourd'hui encore, quelques gémanciens très compétents, mais peu nombreux pratiquent ce genre de divination (par renard ou timba) au Mandé.

Un groupe de Kamara a suivi Suleymane Konaté, mais à cause de diverses contestations existant entre les Konaté et les Kamara, ces derniers voulurent éviter les risques de proximité et se tinrent à distance; dolo, devise des Dogon de la région de Sanga, signifierait "rester à l'écard" en langue ancienne. malinké (1). Au départ, les Konaté avaient pour interdit (tana) la panthère et les Kamara le varan (2) ; il y eut dispersion tout au long du chemin.

Les Tellem, qui ont occupé les falaises avant l'arrivée des Dogon, seraient constitués de groupe émanant de diverses ethnies (dont des Kourouma du Mandé, des Katlé et Ganamé soninké, etc. (3) ; ils s'y étaient réfugiés pour fuir les guerres et

(1) Bien entendu nos informateurs de Sanga ont donné des termes gyindo et dolo, des "étymologies" dogon différentes de celles apportées par Wâ Kamissoko. Voir notamment S. de Ganay, Les Devises des Dogon, Travaux et Mémoires de l'Institut d'Ethnologie, tome XLI 1971 p.9 et 33.

(2) L'interdit de la panthère est rappelé dans la devise des Arou, très long texte dogon que nous avons enregistré, récité lors de l'intronisation du Hogon de cette tribu. Il relate, entre autres, certains épisodes de la migration des Dogon depuis le Mandé. Tous les Kansaye de la région de Bandiagara seraient des Kamara.

(3) Des recherches sont menées depuis plusieurs années par les mem bres de l'Institut d'Anthropologie de l'Université d'Utrecht, notamment par le $\operatorname{Pr}$ J.Huizinga et M. R.Bedaux. Elles ont apporté de précieuses informations sur l'implantation et les restes des Tellem (ossuaires, greniers, habitations) dans la falaise de Bandiagara. Voir R.M.A. Bedaux "Tellem; reconnaissance archéologique d'une culture de l'ouest africain au moyen âge, recherches archéologiques" Journal de la Société des Afri canistes T.XLII, 2, p.103-185. Egalement au même auteur "Tellem, reconnaissance archéologique d'une culture de l'ouest africain au moyen âge : les appuie-nuques" Journal de la sté des africanistes T.XLIV, 1, 1974, p.7-42. 
l'esclavage. Les Mossi actuels descendent en partie de Traoré ayant quitté le Manáe avant l'époque de Soundiata. Il y eut, là aussi, plusieurs vagues de migrations.

Un texte traditionnel en langue malinké qu'il conviendra d'enregistrer, de traduire et de commenter, énumère plus de trois mille noms de clans et de familles, ainsi que l'histoire de leurs implantations géographiques, par régions et par villages, en Afrique Occidentale. 